\title{
Extremely sharp carbon nanocone probes for atomic force microscopy imaging
}

\author{
I-Chen Chen, Li-Han Chen, Xiang-Rong Ye, Chiara Daraio, and Sungho $\mathrm{Jin}^{\mathrm{a})}$ \\ Materials Science and Engineering, University of California, San Diego, California 92093-0411 \\ Christine A. Orme \\ Chemistry and Material Science, Lawrence Livermore National Laboratory, Livermore, California 94550
}

Arjan Quist and Ratnesh Lal

Neuroscience Research Institute, University of California, Santa Barbara, Santa Barbara, California 93106

(Received 25 October 2005; accepted 13 March 2006; published online 10 April 2006)

\begin{abstract}
A simple and reliable catalyst patterning technique combined with electric-field-guided growth is utilized to synthesize a sharp and high-aspect-ratio carbon nanocone probe on a tipless cantilever for atomic force microscopy. A single carbon nanodot produced by an electron-beam-induced deposition serves as a convenient chemical etch mask for catalyst patterning, thus eliminating the need for complicated, resist-based, electron-beam lithography for a nanoprobe fabrication. A gradual, sputtering-induced size reduction and eventual removal of the catalyst particle at the probe tip during electric-field-guided growth creates a sharp probe with a tip radius of only a few nanometers. These fabrication processes are amenable for the wafer-scale synthesis of multiple probes. High resolution imaging of three-dimensional features and deep trenches, and mechanical durability enabling continuous operation for many hours without noticeable image deterioration have been demonstrated. () 2006 American Institute of Physics. [DOI: 10.1063/1.2193435]
\end{abstract}

The key component of atomic force microscopy (AFM) is the probe tip, as the resolution and reliability of AFM imaging is determined by its sharpness, shape, and the nature of materials. Standard commercial probes made of silicon or silicon nitride have tips of a pyramid shape, which do not allow easy access to narrow or deep structural features, and generally have a relatively blunt tip radius on the order of $10 \mathrm{~nm}$. The high-aspect-ratio geometry and excellent mechanical strength of the carbon nanotubes (CNTs) offer advantages for imaging as an AFM tip. Due to their excellent physical and chemical properties, ${ }^{1-3}$ CNTs have been attached onto pyramid tips by various approaches ${ }^{4-8}$ as well as directly grown using thermal chemical vapor deposition (CVD). ${ }^{9-11}$ The attachment methods are manual and time consuming, and often result in nonreproducible CNT configuration and placement. While the thermal CVD approach can potentially lead to the wafer-scale production of AFM tips, the number, orientation, and length of CNTs are difficult to control.

An important aspect to consider in utilizing CNT probes is that the single-walled nanotube probes with a desirable small diameter tend to exhibit an inherent thermal vibration problem if the length is made reasonably long, and hence they cannot be used to trace deep structural profiles. On the other hand, multiwalled nanotubes such as those synthesized in dc plasma enhanced CVD (dc-PECVD) $)^{12-15}$ have a larger diameter in the regime of 20-100 nm and hence exhibit improved mechanical and thermal stability, but the catalyst particle at the nanotube probe tip (or the natural dome structure in a nanotube grown by a base growth mechanism) has a finite radius of curvature, which limits the AFM resolution.

Recently, two approaches have been employed to fabricate multiwalled nanotube probes on tipless cantilevers by

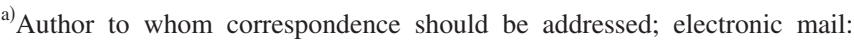
jin@ucsd.edu
}

dc-PECVD. ${ }^{16,17}$ These approaches, however, require somewhat complicated, multiple patterning steps. The catalyst dots in both approaches are patterned by the lift-off of the spin-coated polymethyl methacrylate (PMMA) layer following typical electron- $(e-)$ beam lithography. A reliable and uniform spin coating of a resist layer generally requires a relatively large area, and is difficult to achieve for a tipless cantilever, which has a narrow and elongated geometry. In one of these reports, ${ }^{16}$ patterned catalyst dots were formed before the fabrication of the cantilevers, but the catalyst had to be protected by the PECVD-deposited $\mathrm{Si}_{3} \mathrm{~N}_{4}$ layer in order for the catalyst dots to survive and keep catalytic activity throughout the subsequent microfabrication steps. In the other report, ${ }^{17}$ the e-beam lithography steps had to be used twice to pattern a catalyst dot on the commercial tipless cantilever in order to remove the extra Ni catalyst on the cantilever. The probe tip radii reported are also relatively large.

In this letter, we have fabricated high-aspect-ratio carbon nanocone $(\mathrm{CNC})$ probes with very sharp tips on tipless cantilevers by employing a resist-free e-beam induced deposition (EBID) of carbon masks combined with electric-fieldcontrolled CVD growth. A high resolution AFM imaging of nanoscale features and deep grooves are demonstrated using $\mathrm{CNC}$ probes.

The fabrication process for a CNC probe is schematically illustrated in Fig. 1. First, the top surface of the cantilever (NSC/tipless, MikroMasch, USA) was coated with a $\sim 10 \mathrm{~nm}$ thick Ni film by e-beam evaporation. For the EBID of carbon dots, a JEOL IC845 scanning electron microscope (SEM) with the NPGS software (J. C. Nabity lithography system) was used. The acceleration voltage was $30 \mathrm{kV}$, and the beam current was $50 \mathrm{pA}$. The carbon dot deposition time was varied between 8 and $30 \mathrm{~s}$ depending on the intended size of the dot. No special carbonaceous precursor molecules were introduced as the residual carbon-containing molecules naturally presenting in the chamber were sufficient for the 


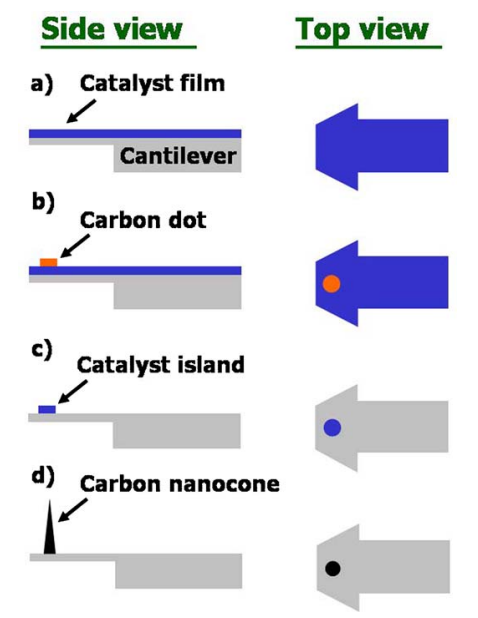

FIG. 1. (Color online) Schematic of the resist-free fabrication technique for a single CNC based AFM tip. (a) Catalyst deposition by e-beam evaporation. (b) E-beam induced deposition of a carbon dot mask. (c) Metal wet etching and the removal of a carbon dot. (d) CVD growth of a CNC probe.

EBID processing to form amorphous carbon dots on the cantilever surface. A single carbon dot with a selected diameter of $\sim 300 \mathrm{~nm}$ was deposited near the front end edge of the cantilever by the EBID as illustrated in Fig. 1(b). The carbon dot serves as a convenient etch mask for chemical etching. The $\mathrm{Ni}$ film was then etched away by using a mixture of $\left[\mathrm{H}_{3} \mathrm{PO}_{4}\right]:\left[\mathrm{HNO}_{3}\right]:\left[\mathrm{CH}_{3} \mathrm{COOH}\right]:\left[\mathrm{H}_{2} \mathrm{O}\right]=1: 1: 1: 2$ except the portion underneath the mask. The removal of the carbon dot mask after the catalyst patterning was performed with an oxygen reactive ion etch (RIE) for $1 \mathrm{~min}$, which exposed the $\mathrm{Ni}$ island as illustrated in Fig. 1(c). The cantilever with the $\mathrm{Ni}$ island was then transferred to the dc-PECVD system for subsequent growth of the CNC, Fig. 1(d). The growth of the $\mathrm{CNC}$ probe was carried out at $700{ }^{\circ} \mathrm{C}$ for $10-20$ min using a mixture of $\mathrm{NH}_{3}$ and $\mathrm{C}_{2} \mathrm{H}_{2}$ gas (ratio 4:1) at 3 mTorr pressure. An applied electric field was utilized to guide the growth of the nanocone along the desired direction.

The EBID of the carbon nanodots is a simple writing technique to directly fabricate nanoscale patterns on the substrate bypassing the use of any e-beam resist layer related steps. ${ }^{18}$ The carbon deposition is caused by the dissociation of the volatile molecules adsorbed on the substrate into a nonvolatile deposit via a high-energy focused electron. Compared with the typical e-beam lithography approach of preparing a single dot pattern on a small cantilever, the EBID process can more accurately pattern the catalytic island at the desired position via in situ control under high magnification of $\times 10000$ or higher in the SEM.

While the use of the EBID carbon patterns have been demonstrated as dry etching masks, ${ }^{18}$ there has been no report for their use as wet etching masks to the best of our knowledge. We investigated the chemical etchability of the carbon dots in various acids and other chemicals such as $\mathrm{HCl}, \mathrm{HF}, \mathrm{HNO}_{3}, \mathrm{H}_{2} \mathrm{O}_{2}$ and acetone, and found that the carbon dots were very stable and remained adherent on the substrate after immersing into these chemicals. The carbon dots have a unique advantage in that while they are resistant to chemical etching, they are easily removable by oxygen RIE. We find that the oxygen RIE process does not affect the $\mathrm{Ni}$ film and reduces its catalytic activity for CNT/CNC nucleation and growth. Experimental studies on CNC growth indicate that the diameter of the $10 \mathrm{~nm}$ thick Ni catalyst island should be kept smaller than $\sim 300 \mathrm{~nm}$ to avoid the undesirDownloaded 28 Sep 2006 to 131.215.225.9. Redistribution subject (a)

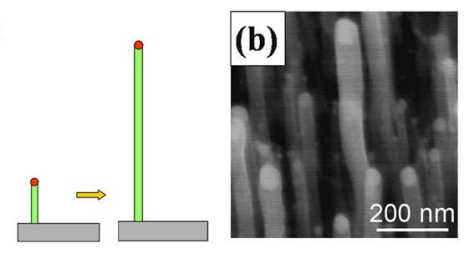

(c)

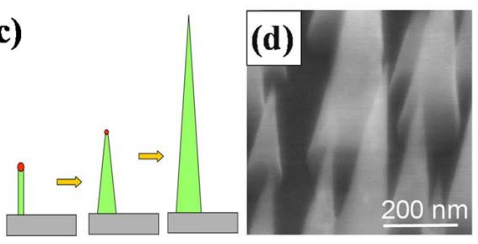

FIG. 2. (Color online) (a) Schematic of an equidiameter carbon nanotube growth. (b) SEM image of equidiameter CNTs grown on an Si substrate at a dc bias of $450 \mathrm{~V}$ for $20 \mathrm{~min}$. (c) Schematic of the gradual reduction of a catalyst particle at the CNT tip by sputtering and accompanying reduction in the CNT diameter. (d) SEM image of the CNC with the catalyst particle completely removed at $550 \mathrm{~V}$ for $20 \mathrm{~min}$.

able nucleation and growth of multiple CNCs. Our carbon island chemical etch mask technique is generally useful for creating a pattern on any small samples such as a prefabricated tipless cantilever, on which the resist layer cannot be uniformly coated for reliable lithography.

By adjusting the applied bias in the dc-PECVD system, the morphology of CNTs can be controlled. At a low applied voltage of $450 \mathrm{~V}$, the size of the catalyst particles does not change during growth and the resultant CNTs are equidiameter nanotubes as shown in Figs. 2(a) and 2(b). When the applied voltage is increased, e.g., $550 \mathrm{~V}$, the diameter of the catalyst particle on the tip can be gradually reduced due to a plasma etching (sputtering) effect, as indicated in Figs. 2(c) and 2(d). The gradually diminishing catalyst size causes the nanotube diameter to change with the growth time, resulting in a nanocone configuration and the eventual complete elimination of the catalyst particle at the CNC tip, which is the key mechanism to obtain a very sharp tip.

Figures 3(a) and 3(b) show the SEM images of our CNC probe (marked by an arrow) grown on a tipless cantilever. In Fig. 3(a), a single CNC probe grown near the edge of a tipless cantilever is shown. Figure $3(\mathrm{~b})$, a higher magnification SEM micrograph, shows a CNC with $\sim 2.5 \mu \mathrm{m}$ height, $200 \mathrm{~nm}$ base diameters, and a cone angle $<5^{\circ}$. The inset in Fig. 3(b) is an example transmission electron microscopy (TEM) image of a CNC tip, which shows the tip radius of the curvature of only a few nanometers. The microstructure of the nanocone appears to be a mixture of crystalline and amorphous phases in general agreement with previous work. ${ }^{13,19}$ It should be noted that the CNC probe in Fig. 3(b) is made intentionally tilted by manipulating the electric field direction during CVD growth. Such a tilted probe is desir-

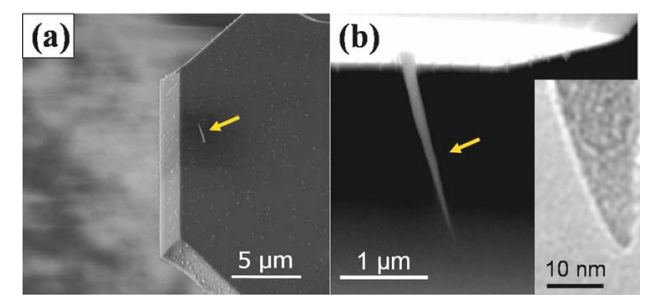

FIG. 3. (Color online) (a) Top view SEM image of the single CNC probe grown near the edge of a cantilever (low-magnification, $30^{\circ}$ tilted view). (b) Side view SEM image of the CNC probe. Inset: TEM image of the CNC tip. o AlP license or copyright, see http://apl.aip.org/apl/copyright.jsp 
(a)

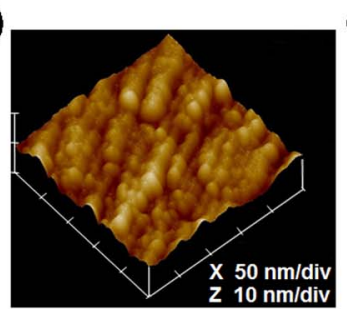

(b)

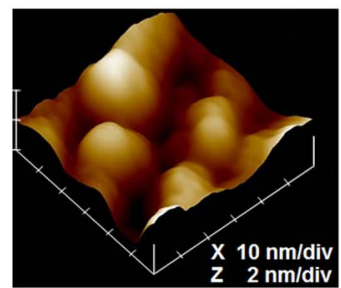

(c)

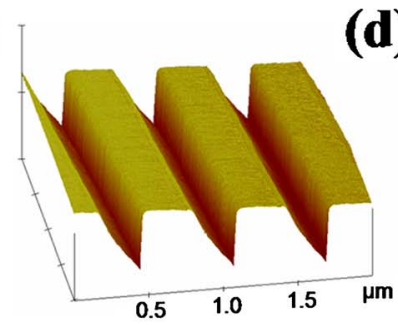

(d)

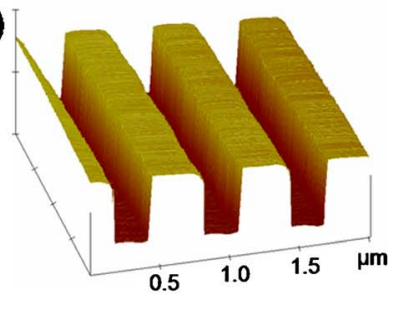

(e)

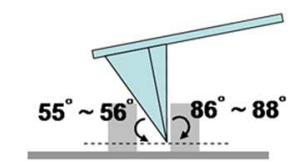

(f)

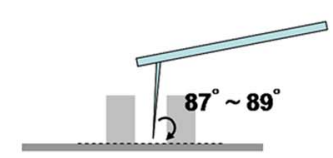

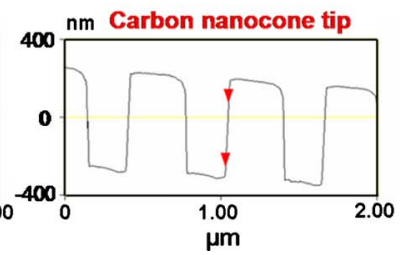

FIG. 4. (Color online) (a) Copper film AFM image. (b) Zoom-in Cu film AFM image. (c) AFM image of a PMMA line pattern by a conventional $\mathrm{Si}$ pyramid tip. (d) Image of the same PMMA pattern by a CNC tip. (e) The height profile of image (c). (f) The height profile of image (d).

able as it compensates the operation tilt angle of the AFM cantilever so that the probe itself is close to being vertical for stable imaging. The tilt angle of the $\mathrm{CNC}$ probe is $\sim 13^{\circ}$ with respect to the normal direction of the cantilever surface.

The performance of the $\mathrm{CNC}$ probe was evaluated in the tapping mode using a Dimension 3100 AFM with a Nanoscope IIIa controller (VEECO Instruments) for imaging in air. The surface of a copper film $(\sim 300 \mathrm{~nm}$ thick $)$ sputter deposited on the Si surface was imaged by using our CNC probe, as shown in Figs. 4(a)-4(c). These images clearly show a well-defined and rounded grain structure, even for the grain size of $\sim 5 \mathrm{~nm}$ or smaller. The sharper grain boundaries and image quality were well revealed due to the sharpness of the CNC tip.

To demonstrate the advantage of the high aspect ratio of a CNC tip, a $300 \mathrm{~nm}$ line/space, $500 \mathrm{~nm}$ deep PMMA pattern was evaluated and the imaging performance of the conventional Si tip versus our CNC tip was compared. The image acquired with a Si probe, Fig. 4(c), shows a misleading image pattern due to the angle of the pyramid. The $\mathrm{CNC}$ probe, on the other hand, reveals the true geometry of the pattern including the configuration of the vertical walls implying that the CNF probe is able to trace the contour of the deep profile pattern, as presented in Fig. 4(d). From Fig. 4(e), the imaged right sidewall slope of $55^{\circ}-56^{\circ}$ and a left sidewall slope of $86^{\circ}-88^{\circ}$ of the pattern actually matches those of the Si pyramid shape itself. From a calculation of geometry, the Si pyramid tip cannot possibly reach the bottom of the PMMA pattern. The experimental results also confirmed this. As shown in Fig. 4(f), the sidewall slopes of the same pattern

acquired with the $\mathrm{CNC}$ probe are about $87^{\circ}-89^{\circ}$. These data demonstrate that the $\mathrm{CNC}$ probe is is strong enough to trace the abrupt step structure without breakage.

In order to evaluate the mechanical durability and adhesion strength of the $\mathrm{CNC}$ probe, the probe was operated on a continuous scan mode on $\mathrm{Au}$ or $\mathrm{Cu}$ film samples for as long as $8 \mathrm{~h}$. The lateral resolution of the obtained AFM image was not noticeably changed as compared to the initially scanned image at time zero (data not shown).

In summary, the fabrication of a sharp and high-aspectratio carbon nanocone probe that possesses desirable thermal stability and mechanical toughness has been demonstrated using resist-free patterning of catalyst nanodots and electric field guided CVD growth. The catalyst particle on the nanocone tip was completely removed via time-dependent size reduction, thus leading to an extremely sharp tip, which can be used for AFM imaging and deep profile analysis.

The authors acknowledge the support of the work by NSF-NIRTs under Grant Nos. DMI-0210559 and DMI0303790, UC Discovery Fund under Grant No. ele02-10133/ Jin, Lawrence Livermore National Lab (LLNL) MRI Fund Contract No. 04-006, National Institute of General Medical Sciences (Contract No. GM056290), the Philip Morris External Grant Program, and Alzheimer's Disease Program of California Department of Health, U.S. Department of Energy by the University of California via Lawrence Livermore National Laboratory under Contract No. W-7405-Eng-4.

${ }^{1}$ M. S. Dresselhaus, G. Dresselhaus, and Ph. Avouris, editors Carbon Nanotubes: Synthesis, Structure, Properties, and Applications (Springer, Berlin, 2001).

${ }^{2}$ C. Bower, W. Zhu, D. Shalom, D. Lopez, L. H. Chen, P. L. Gammel, and S. Jin, Appl. Phys. Lett. 80, 3820 (2002).

${ }^{3}$ A. Fennimore, T. Yuzvinsky, W. Han, M. Fuhrer, J. Cumings, and A. Zettl, Nature (London) 424, 408 (2003).

${ }^{4}$ H. Dai, J. H. Hafner, A. G. Rinzler, D. T. Colbert, and R. E. Smalley, Nature (London) 384, 147 (1996).

${ }^{5}$ H. Nishijima, S. Kamo, S. Akita, Y. Nakayama, K. I. Hohmura, S. H. Yoshimura, and K. Takeyasu, Appl. Phys. Lett. 74, 4061 (1999).

${ }^{6}$ R. Stevens, C. Nguyen, A. Cassell, L. Delzeit, M. Meyyappan, and J. Han, Appl. Phys. Lett. 77, 3453 (2000).

${ }^{7}$ A. Hall, W. G. Matthews, R. Superfine, M. R. Falvo, and S. Washburna, Appl. Phys. Lett. 82, 2506 (2003).

${ }^{8}$ J. Tang, G. Yang, Q. Zhang, A. Parhat, B. Maynor, J. Liu, L. C. Qin, and O. Zhou, Nano Lett. 5, 11 (2005).

${ }^{9}$ J. H. Hafner, C. L. Cheung, and C. M. Lieber, Nature (London) 398, 761 (1999).

${ }^{10}$ C. L. Cheung, J. H. Hafner, T. W. Odom, K. Kim, and C. M. Lieber, Appl. Phys. Lett. 76, 3136 (2000).

${ }^{11}$ E. Yenilmez, Q. Wang, R. J. Chen, D. Wang, and H. Dai, Appl. Phys. Lett. 80, 2225 (2002).

${ }^{12}$ V. I. Merkulov, A. V. Melechko, M. A. Guillorn, M. L. Simpson, D. H. Lowndes, J. H. Whealton, and R. J. Raridon, Appl. Phys. Lett. 80, 4816 (2002).

${ }^{13}$ L.-H Chen, J. F. AuBuchon, A. Gapin, C. Daraio, P. Bandaru, S. Jin, D. W. Kim, I. K. Yoo, and C. M. Wang, Appl. Phys. Lett. 85, 5373 (2004).

${ }^{14}$ J. F. AuBuchon, L. H. Chen, A. I. Gapin, D. W. Kim, C. Daraio, and S. Jin, Nano Lett. 4, 1781 (2004).

${ }^{15}$ M. Chhowalla, K. B. K. Teo, C. Ducati, N. L. Rupesinghe, G. A. J. Amaratunga, A. C. Ferrari, D. Roy, J. Robertson, and W. I. Milne, J. Appl. Phys. 90, 5308 (2001).

${ }^{16}$ Q. Ye, A. M. Cassell, H. B. Liu, K. J. Chao, J. Han, and M. Meyyappan, Nano Lett. 4, 1301 (2004).

${ }^{17}$ H. Cui, S. V. Kalinin, X. Yang, and D. H. Lowndes, Nano Lett. 4, 2157 (2004).

${ }^{18}$ A. N. Broers, W. W. Molzen, J. J. Cuomo, and N. D. Wittels, Appl. Phys. Lett. 29, 596 (1976).

${ }^{19}$ W. H. Wang, Y. T. Lin, and C. T. Kuo, Diamond Relat. Mater. 14, 907 (2005) 